\title{
Early experience with a novel net temporary bridging device (Cascade) to assist endovascular coil embolization of intracranial aneurysms
}

\author{
Alejandro Tomasello, MD, 1,2 David Hernandez, MD,,2 Laura Ludovica Gramegna, MD, ,2,3 \\ Sonia Aixut, MD, ${ }^{4}$ Roger Barranco Pons, MD, ${ }^{4}$ Olav Jansen, MD, ${ }^{5}$ Michal Zawadzki, MD, PhD, ${ }^{6}$ \\ Antonio Lopez-Rueda, MD, ${ }^{7}$ Carmen Parra-Fariñas, MD, ${ }^{2}$ Carlos Piñana, MD, ${ }^{1}$ Lavinia Dinia, PhD, ${ }^{1,2}$ \\ Fuat Arikan, MD, PhD, ${ }^{8}$ and Alex Rovira, MD ${ }^{2,9}$ \\ ${ }^{1}$ Interventional Neuroradiology Section, Department of Radiology, Vall d'Hebron University Hospital, Barcelona; ${ }^{2} V a l l ~ d ' H e b r o n$ \\ Research Institute (VHIR), Vall d'Hebron University Hospital, Barcelona, Spain; ${ }^{3}$ RCCS Istituto delle Scienze Neurologiche di \\ Bologna, Italy; ${ }^{4}$ Department of Neuroradiology, Hospital Universitari de Bellvitge de Llobregat, Barcelona, Spain; ${ }^{5}$ Department \\ of Radiology and Neuroradiology, University Hospital Schleswig-Holstein, Campus Kiel, Germany; ${ }^{6}$ Division of Interventional \\ Neuroradiology, Department of Radiology, Centre of Postgraduate Medical Education, Warsaw, Poland; ${ }^{7}$ Department of \\ Radiology, Hospital Clinic I Provincial de Barcelona; ${ }^{8}$ Department of Neurosurgery, Vall d'Hebron University Hospital, and \\ Neurotraumatology and Neurosurgery Research Unit (UNINN), Vall d'Hebron Research Institute, Barcelona; and ${ }^{9}$ Section of \\ Neuroradiology and Magnetic Resonance Unit, Vall d'Hebron University Hospital, Barcelona, Spain
}

OBJECTIVE The goal of this study was to evaluate the effectiveness and safety of a new noncompletely occlusive netassisted remodeling technique in which the Cascade net device is used for temporary bridging of intracranial aneurysms. METHODS Between July 2018 and May 2019, patients underwent coil embolization with the Cascade net device within 4 centers in Europe. Analysis of angiographic (modified Raymond-Roy classification [MRRC]) and clinical outcomes data was conducted immediately following treatment and at the 6-month follow-up.

RESULTS Fifteen patients were included in the study (mean age $58 \pm 13$ years, $11 / 15$ [73.3\%] female). Ten patients had unruptured aneurysms, and 5 presented with ruptured aneurysms with acute subarachnoid hemorrhage. The mean aneurysm dome length was $6.27 \pm 2.33 \mathrm{~mm}$ and the mean neck width was $3.64 \pm 1.19 \mathrm{~mm}$. Immediately postprocedure, MRRC type I (complete obliteration) was achieved in 11 patients (73.3\%), whereas a type II (residual neck) was achieved in 4 patients (26.7\%). Follow-up examination was performed in 7/15 patients and showed stabilization of aneurysm closure with no thromboembolic complications and only 1 patient with an increased MRRC score (from I to II) due to coil compression.

CONCLUSIONS Initial experience shows that the use of a new noncompletely occlusive net-assisted remodeling technique with the Cascade net device may be safe and effective for endovascular coil embolization of intracranial aneurysms.

https://thejns.org/doi/abs/10.3171/2019.11.JNS192477

KEYWORDS intracranial aneurysm; endovascular procedures; coil embolization; Cascade remodeling net; fibrinolytic agents; vascular disorders; interventional neurosurgery

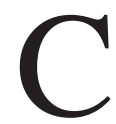
OIL embolization of intracranial aneurysms with complex anatomy, such as wide-necked aneurysms, is challenging and requires the use of adjunctive devices to provide coil stabilization and avoid loop herniation. ${ }^{7}$ The balloon catheter-assisted remodeling technique (BRT) has improved the approach to coil embolization of intracranial aneurysms..$^{12}$ To perform the
BRT, a balloon catheter is positioned before the aneurysm neck during embolization to assist the insertion of coils into the sac, avoiding herniation. A multicenter prospective study showed that ruptured aneurysms treated with the assistance of the BRT have better postoperative occlusion rates than those treated without it $(94.9 \%$ vs $88.7 \%$, $\mathrm{p}=0.017)$, with no increase in mortality and morbidity. ${ }^{11}$

ABBREVIATIONS BRT = balloon catheter-assisted remodeling technique; DSA = digital subtraction angiography; ICA = internal carotid artery; MRRC = modified Raymond-Roy classification; $\mathrm{mRS}$ = modified Rankin Scale; SCA = superior cerebellar artery; VA = vertebral artery.

SUBMITTED September 12, 2019. ACCEPTED November 18, 2019.

INCLUDE WHEN CITING Published online January 24, 2020; DOI: 10.3171/2019.11.JNS192477. 


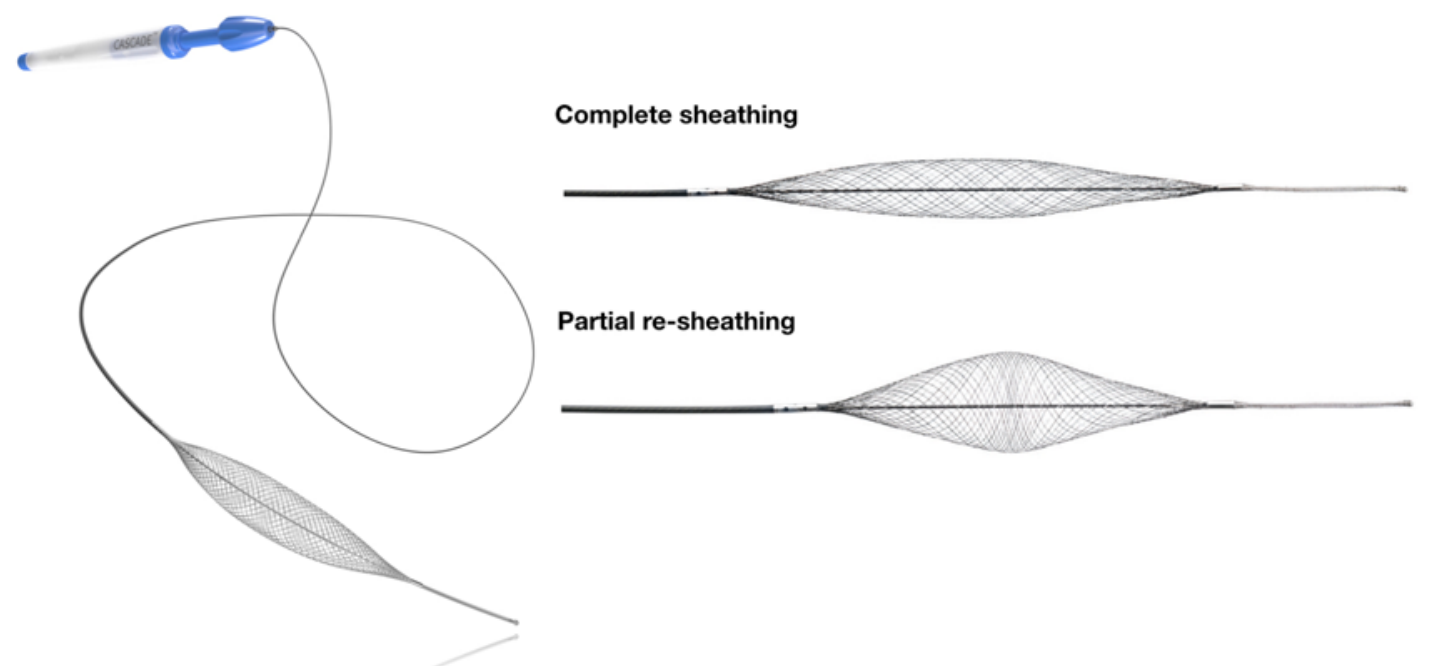

FIG. 1. Cascade net device. Left: The remodeling-net Cascade device with handle. Right: Images illustrating the option for sheathing the braided net and partially unsheathing it, achieving a straight noncontorted shape. Figure is available in color online only.

However, this technique creates temporary flow arrest in the parent artery, which may stress the clinician and potentially increase procedural risk for thromboembolic complications, even if this has not been confirmed in randomized studies. ${ }^{11,13}$

To overcome these challenges, the net-assisted remodeling technique has been proposed,, 9, in which a braided net temporarily bridges the aneurysm neck to support coil embolization without compromising flow in the parent artery. The first device created for this purpose was the Comaneci device (Rapid Medical). ${ }^{6}$ Our study aimed to present initial multicenter clinical experience to evaluate the effectiveness and safety of the net-assisted remodeling technique with a new nonocclusive Cascade net device (Perflow Medical), which has a braided net with a cell size of $0.3 \mathrm{~mm}^{2}$; this is significantly smaller than the currently available coils used for intracranial aneurysm embolization and it is fully removable at the end of the procedure. The device's architecture protects the parent artery during coil embolization without flow arrest in the distal vasculature, while avoiding the entanglement of coils.

\section{Methods \\ Patient Population}

We recruited all consecutive patients between July 2018 and May 2019 in whom the remodeling technique with a balloon catheter was deemed necessary to assist endovascular coil embolization of intracranial aneurysms. The decision to use the remodeling technique was made at the discretion of the interventional neuroradiologist. We treated with a net temporary bridging device (Cascade) all consecutive large-necked aneurysms and every challenging situation in which a remodeling technique would be deemed necessary according to the interventional neuroradiologist's decision. Patients older than 18 years of age with a ruptured or unruptured saccular aneurysm located in the anterior or posterior circulation with no previous stent implantation in the target vascular territory were included. Patients with an unruptured aneurysm were informed about the planned treatment strategy and were provided a detailed explanation of all treatment alternatives at least 24 hours before the procedure. An informed consent was obtained from patients with a ruptured aneurysm immediately before the procedure.

\section{Cascade Net Device}

The Cascade dynamic noncompletely occlusive remodeling net (Cascade net; Perflow Medical) is a CE-marked, radiopaque, adjustable braided net composed of nitinol and platinum wires, which provide mechanical support during coil embolization of intracranial aneurysms. It is fully radiopaque to achieve optimum visualization. The maximal cell size is $0.3 \mathrm{~mm}^{2}$, which is smaller than the diameter of the secondary structure of any currently available coils. The diameter of the braided net ranges between 0.5 and $6 \mathrm{~mm}$, with length measuring between markers of $37 \mathrm{~mm}$ when fully deflated and $10 \mathrm{~mm}$ when fully inflated. The diameter and length are adjustable by the operator via a handle that provides an expected straight foreshortening of the device and adaptation of the radial force in order to achieve optimal aneurysm neck coverage (Fig. 1). Two models of the device are available: The $\mathrm{M}$ model has an effective braid diameter of $0.5-4 \mathrm{~mm}$ and is recommended for vessel diameters of $2-4 \mathrm{~mm}$. The $\mathrm{L}$ model has an effective braid diameter of $0.5-6 \mathrm{~mm}$ and is recommended for vessel diameters of 4-6 mm. Both models are compatible with a 0.021 -inch microcatheter and have a distal tip wire, allowing for gentle and safe navigation.

\section{Antiplatelet and Anticoagulant Regimen}

All patients received full heparinization (3000-6000 IU) during the procedure. Patients with an unruptured aneurysm $(n=10)$ were given the following antiplatelet/anticoagulant regimen: 6 received aspirin $(500 \mathrm{mg})$ before or 
during the procedure (all were considered preprocedure); 1 received a bolus of inyesprin $(900 \mathrm{mg})$ during treatment; 6 received dual-antiplatelet therapy (aspirin $100 \mathrm{mg}$ daily, clopidogrel $75 \mathrm{mg}$ daily) for $10(\mathrm{n}=5)$ or $12(\mathrm{n}=1)$ days postprocedure; 1 received aspirin (150 mg daily for 1 month); and 1 received clopidogrel (150 $\mathrm{mg}$ for 10 days).

Patients with a ruptured aneurysm $(n=5)$ were given the following antiplatelet/anticoagulant regimen: 3 received a bolus of inyesprin (none of the 3 received postprocedural dual-antiplatelet therapy), and 2 received only heparinization (Table 1). No patient received long-term postprocedural antiaggregation therapy.

\section{Endovascular Procedures}

Either a 7-Fr guiding catheter (Envoy; DePuy Synthes) or a 6-Fr long sheath (NeuronMax; Penumbra, Inc.) was used to catheterize the internal carotid artery (ICA) or vertebral artery (VA). After performing 3D rotational angiography, a Rebar 18 catheter (Medtronic, Inc.) was navigated into the parent vessel distally to the aneurysm, while an Excelsior SL10 (Stryker Neurovascular), Echelon 10 (Medtronic, Inc.), or Echelon 14 (Medtronic, Inc.) microcatheter was positioned inside the aneurysm. The diameter of the parent artery determined the size of the Cascade net used. After the device was fully unsheathed and the braid expanded, the Cascade net was moved backward slightly and then forward, partially resheathing the net until the operator was fully satisfied with the device location and the best neck coverage possible.

Next, the first coil was inserted into the aneurysm with the microcatheter inside the sac. The Cascade net was deflated before detachment of the last coil to confirm stability of the coils inside the aneurysm. Finally, the Cascade net was retrieved after removal of the intrasaccular microcatheter.

\section{Outcome and Follow-Up}

Aneurysm characteristics were recorded, including rupture status, location, neck width $(\mathrm{mm})$, dome length $(\mathrm{mm})$, and dome/neck ratio. The number of coils inserted, duration of the Cascade net usage, type of complications, and aneurysm occlusion, measured by the modified Raymond-Roy classification (MRRC) of type I (complete obliteration) or type II (residual neck) ${ }^{16}$ were assessed immediately postprocedure by senior operators with more than 10 years of experience. A complete neurological examination was performed after the procedure in all patients on awakening, at discharge, and at the 6-month follow-up for those who received angiographic follow-up $(n=7)$. Seven patients underwent angiographic follow-up with digital subtraction angiography (DSA) examination after 6 months, no follow-up occurred for 5 patients due to time restrictions, and 3 declined the angiographic follow-up because of good clinical condition. The modified Rankin Scale (mRS) score was recorded at 3 months, as the standard of care in our hospital.

\section{Statistical Analysis}

Statistical analysis was conducted using GraphPad Prism software (version 7; GraphPad Software, Inc.). De- scriptive analysis included frequencies and percentages for categorical variables and mean (SD) or median (IQR) for continuous variables. Fisher's exact test was performed to determine the statistical significance of differences in categorical data for subgroups, and values with $\mathrm{p}<0.05$ were considered significant.

\section{Results}

\section{Patient and Aneurysm Characteristics}

Fifteen patients were included in the study (mean age $58 \pm 13$ years, 11 [73.3\%] female); 10 harbored unruptured aneurysms, and 5 presented with ruptured aneurysms with subarachnoid hemorrhage (Tables 1 and 2). Patient and aneurysm characteristics are presented in Table 1 . The mean aneurysm dome length was $6.27 \pm 2.33 \mathrm{~mm}$ and the mean neck width was $3.64 \pm 1.19 \mathrm{~mm}$. Thirteen patients presented with an aneurysm in the anterior circulation: 1 in the cavernous segment of the ICA; 3 in the paraophthalmic segment of the ICA; 2 on the ICA bifurcation; and 7 in the posterior communicating segment of the ICA. Two patients presented with an aneurysm in the posterior circulation: 1 on the left superior cerebellar artery (SCA) origin (Fig. 2) and 1 on the $\mathrm{V}_{4}$ segment of the right VA (Fig. 3 ). Of the 15 total aneurysms, 11 were wide-necked lesions (dome/neck ratio $\leq 2$ ), whereas 4 were small-necked lesions (dome/neck ratio $>2$ ).

\section{Technical Results}

Procedural characteristics and outcomes are presented in Tables 1 and 2. In all cases the device was successfully positioned, with preservation of distal flow. Immediately postprocedure, MRRC type I was achieved in 11/15 (73.3\%) aneurysms (Fig. 4) and type II in 4/15 (26.7\%). Figures 2-4 show pre- and postprocedure imaging from 3 illustrative cases. In 1 patient a small neck was intentionally left to avoid occluding the parent artery with a large number of coils at the neck because of the parent vessel's small diameter (Fig. 2). The mean number of coils inserted was $5 \pm 2$. The mean duration of Cascade net usage was $30 \pm 14$ minutes. Seventy-five coils were inserted with no entanglement or late protrusion. No thromboembolisms or aneurysm ruptures occurred during the endovascular intervention. DSA examinations performed immediately following the procedure and at follow-up were analyzed for rupture status, aneurysm location (anterior or posterior circulation), and dome/neck ratio. However, none of these factors had any significant effect on the MRRC (type I or II) following endovascular intervention $(p>0.05)$.

\section{Complications}

There were no coil herniations or entanglements during the procedures. Clots were not observed in the internal or external part of the device's net during postprocedure angiographic examination or visual examination of the device on removal. There were no clinical or angiographic complications during treatment. All patients with unruptured aneurysms were discharged, per the sites' standard of care procedures, with $\mathrm{mRS}$ score 0 , and there were no complications associated with the postprocedure antiplatelet regimen. Patients with ruptured aneurysms were 


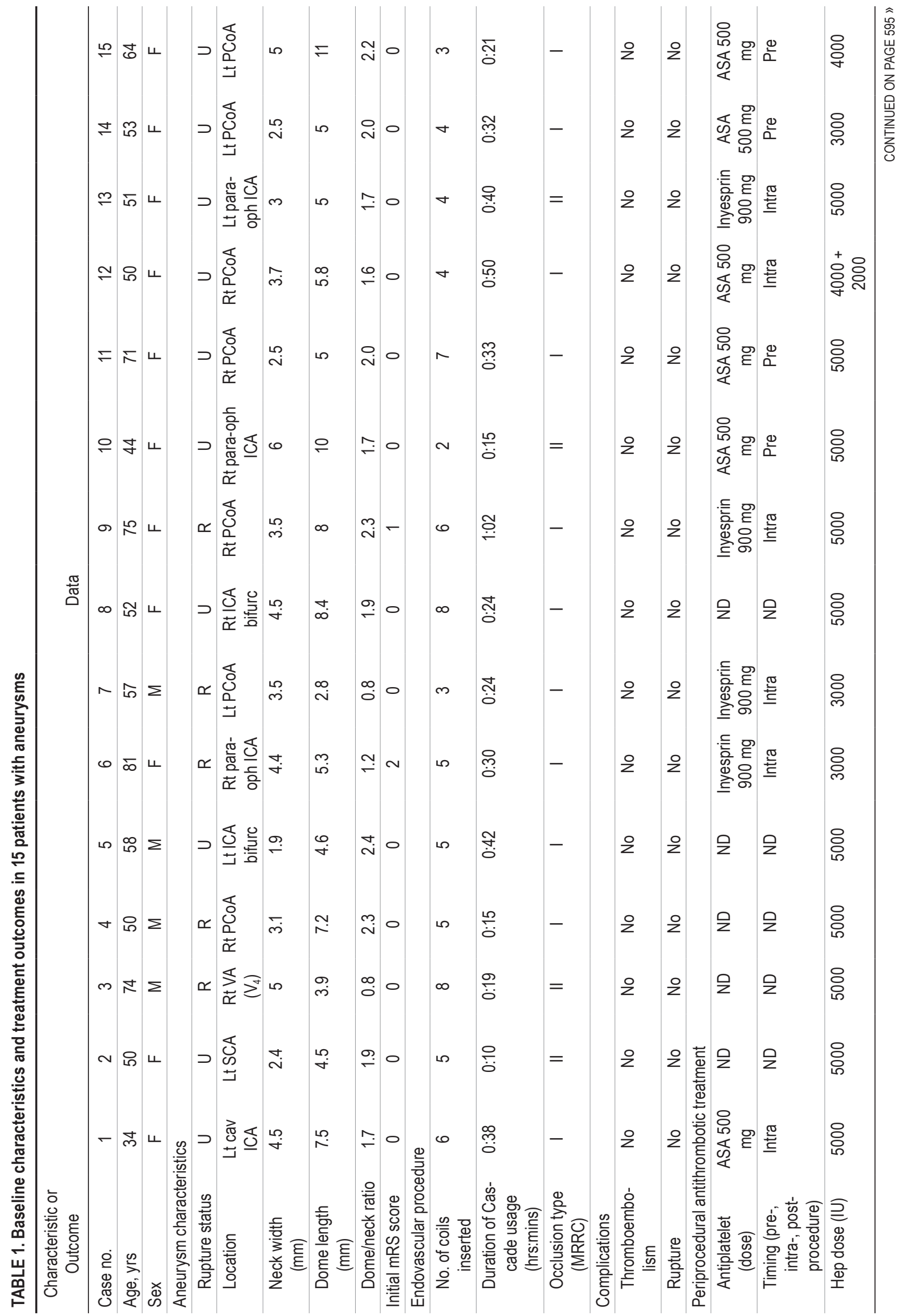




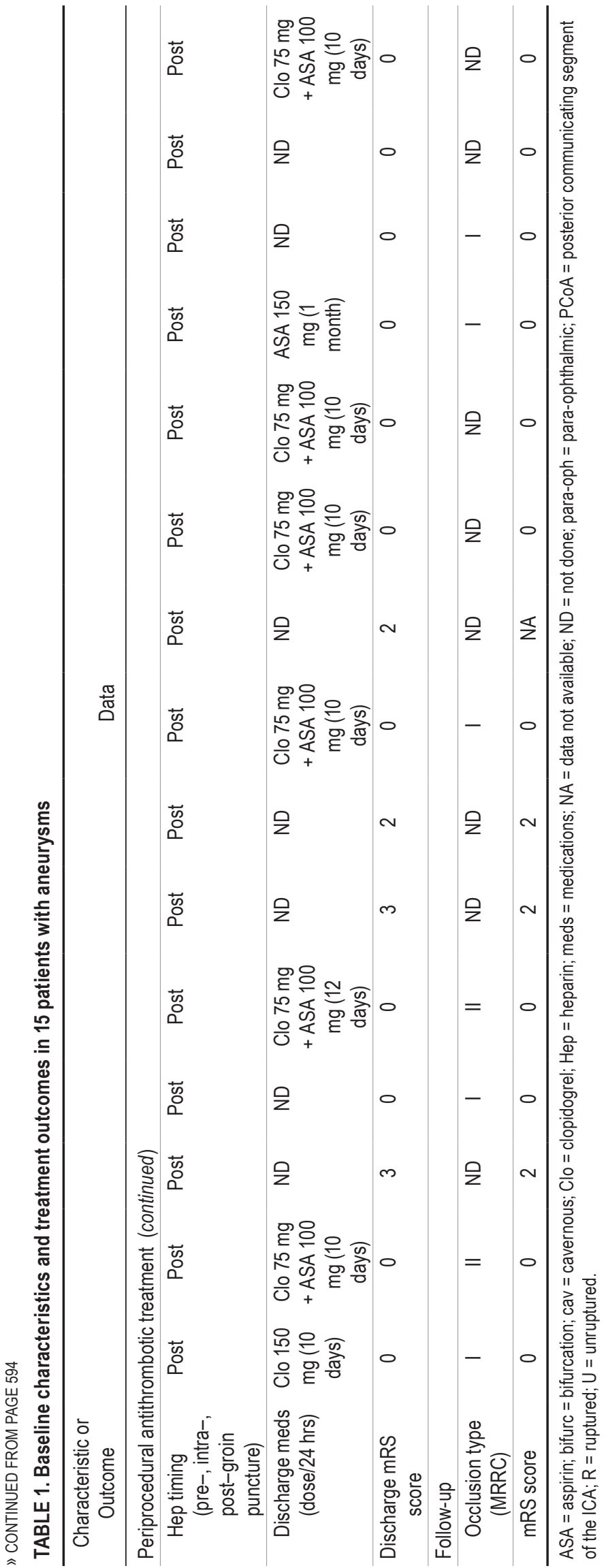

discharged with mRS 0 in 1 patient, mRS 2 in 2 patients, and $\mathrm{mRS} 3$ in 2 patients.

\section{Follow-Up}

Seven of 15 (46.7\%) patients received angiographic follow-up with DSA examination after 6 months. Although complete occlusion was maintained in 5/6 (83.3\%) patients, 1 patient (case 5) developed a residual neck at follow-up despite having immediate complete occlusion. Notably, the patient in case 3 improved from having a residual neck immediately following intervention to complete occlusion at follow-up. Like the immediate outcome analysis, postendovascular intervention, condition and location of aneurysm, and dome/neck ratio had no significant effects on the MRRC. For the patient in case 2, in whom a small neck was intentionally left uncovered, it remained unchanged (Fig. 2). In fact, the patient in case 2 harbored a relatively large aneurysm (dome length 4.5 $\mathrm{mm}$ ) in a very small artery, the SCA; therefore, the treating physician decided to achieve an MRRC type II at the end of the procedure, to prevent any risk of occluding the small parent artery. This level of occlusion was maintained at follow-up. At follow-up, patients did not demonstrate a modification of clinical status or occurrence of bleeding. For patients with ruptured aneurysms, mRS scores at the 3 -month follow-up were mRS 0 for 1 patient, mRS 2 for 3 patients, and 1 patient had no mRS data available due to time restrictions.

\section{Discussion}

We present our preliminary experience performing a remodeling-net technique with a new temporary bridging device (Cascade net) that assists coil embolization of intracranial aneurysms with low risk of coil entanglement. This technique is noncompletely occlusive and allows continuous blood flow in the parent vessel during the procedure, lessening time pressure to the neurointerventionalist related to the risk of ischemia and thromboembolism. The device was successfully inserted in all cases with no procedural complications. Collectively, these data suggest that treatment with the Cascade net device may be effective and safe, with no rupture or thromboembolic complications in our series.

We observed complete occlusion (MRRC type I) in $73.3 \%$ of cases, which is in line with the results of aneurysm embolization with standard coiling in the large case series. ${ }^{11}$ Notably, dome/neck ratios $\leq 2$ did not have worse occlusion outcomes. One patient harboring an aneurysm $<3 \mathrm{~mm}$ was successfully treated; this finding is of interest given that endovascular coiling of small intracranial aneurysms is challenging due to the difficulty of obtaining a stable microcatheter position, and because small aneurysms are more prone to rupture. ${ }^{2}$ Our results suggest that the device can effectively occlude both wide-necked and small aneurysms, although this result requires further investigation with a larger group of patients.

The first description of BRT was published by Moret et al. in $1997^{10}$ and involved temporary inflation of a nondetachable balloon in front of the aneurysm neck during coil placement. A major limitation of BRT is the need for 
Tomasello et al.

TABLE 2. Intraprocedural complications and radiological outcomes in 15 patients with aneurysms

\begin{tabular}{cccccc}
\hline \multirow{2}{*}{ Outcome } & \multicolumn{2}{c}{ Rupture Status } & & \multicolumn{2}{c}{ Dome/Neck Ratio } \\
\cline { 2 - 3 } \cline { 6 - 6 } & Ruptured, $\mathrm{n}=5$ & Unruptured, $\mathrm{n}=10$ & & $\leq 2, \mathrm{n}=11$ & $>2, \mathrm{n}=4$ \\
\hline Complications & $0 / 5(0 \%)$ & $0 / 10(0 \%)$ & & $0 / 11(0 \%)$ & $0 / 4(0 \%)$ \\
\hline Immediate MRRC I & $4 / 5(80 \%)$ & $7 / 10(70 \%)$ & & $7 / 11(63.6 \%)$ & $4 / 4(100 \%)$ \\
\hline Immediate MRRC II & $1 / 5(20 \%)$ & $3 / 10(30 \%)$ & & $4 / 11(36.4 \%)$ & $0 / 4(0 \%)$ \\
\hline Follow-up MRRC I & $4 / 6(66.7 \%)$ & $1 / 1(100 \%)$ & & $4 / 5(80 \%)$ & $1 / 2(50 \%)$ \\
\hline Follow-up MRRC II & $2 / 6(33.3 \%)$ & $0 / 1(0 \%)$ & & $1 / 5(20 \%)$ & $1 / 2(50 \%)$ \\
\hline
\end{tabular}

flow arrest and the risk of thromboembolism. In that original series, 52 wide-necked or complex-shaped aneurysms were treated successfully with BRT, and angiographic evidence of clotting was observed during the procedure in 3 cases, resulting in 1 permanent deficit (quadrantanopia). ${ }^{10}$ Subsequently, 2 prospective studies have evaluated the safety of the BRT in comparison to standard coiling and found that the incidence of thromboembolic events was not statistically higher in patients treated with BRT. The ATENA (Analysis of Treatment by Endovascular Approach of Nonruptured Aneurysms) study was a consecutive series of 547 patients with 572 unruptured aneurysms treated either with the BRT or with coiling alone, and it was found that thromboembolic events occurred in 5.4\% in the BRT group and in $6.2 \%$ in the coiling group with no statistically significant difference. ${ }^{13}$ In the CLARITY (Clinical and Anatomic Results in the Treatment of Rup- tured Intracranial Aneurysms) study ${ }_{11}^{11}$ a consecutive series of 768 patients with ruptured aneurysms, the rate of thromboembolic events was not statistically different in patients treated with either the remodeling technique or coiling alone. Additional studies have evaluated whether total balloon inflation time, the number of inflations, or a minimum effective reperfusion time between inflations increases the risk for thromboembolism, assessed via diffusion-weighted MRI. ${ }^{1,15}$ In both studies, none of these factors correlated with thromboemboli formation and only higher maximum inflation time was significantly correlated to watershed pattern infarcts.

In our case series we found no evidence of thromboembolism either angiographically or clinically. This may be related to the noncompletely occlusive characteristics of the device that allows for a continuous blood flow in the parent vessel during the procedure with no flow arrest
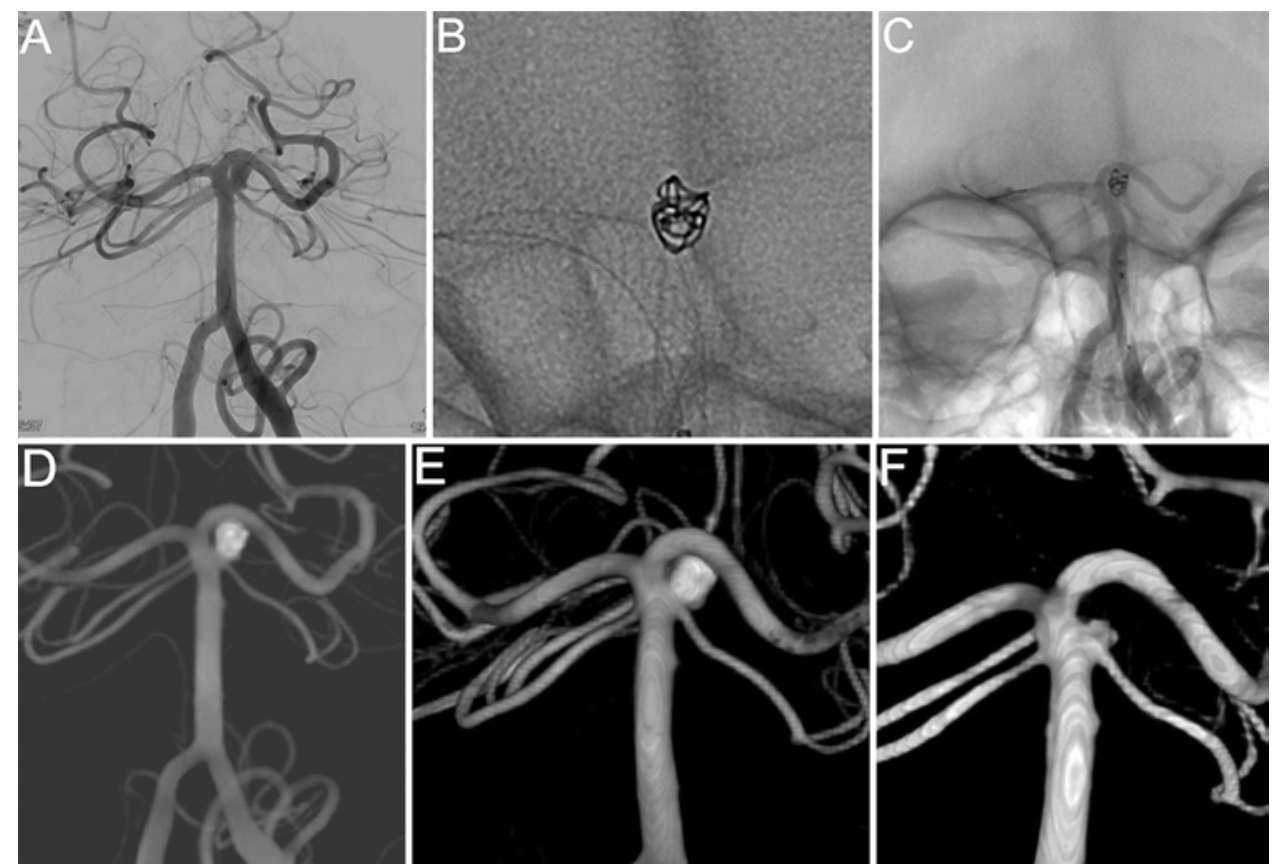

FIG. 2. Case 2. Angiographic imaging of an SCA aneurysm. A: Preprocedural DSA (posterior-anterior view) showing an aneurysm in the left SCA origin. B: Unsubtracted imaging of Cascade placement in the parent vessel and coiling of the aneurysm sac. C: Intraprocedural DSA (posterior-anterior view) showing coils in the aneurysm. D: Postprocedural DSA showing near-complete occlusion of the aneurysm (MRRC type II), with a small neck left to avoid risk of occluding the small patent artery. E and F: 3D rotational angiography taken at the 6 -month follow-up showing stability of the occlusion without $(E)$ and with $(F)$ suppression of the coil signal. 

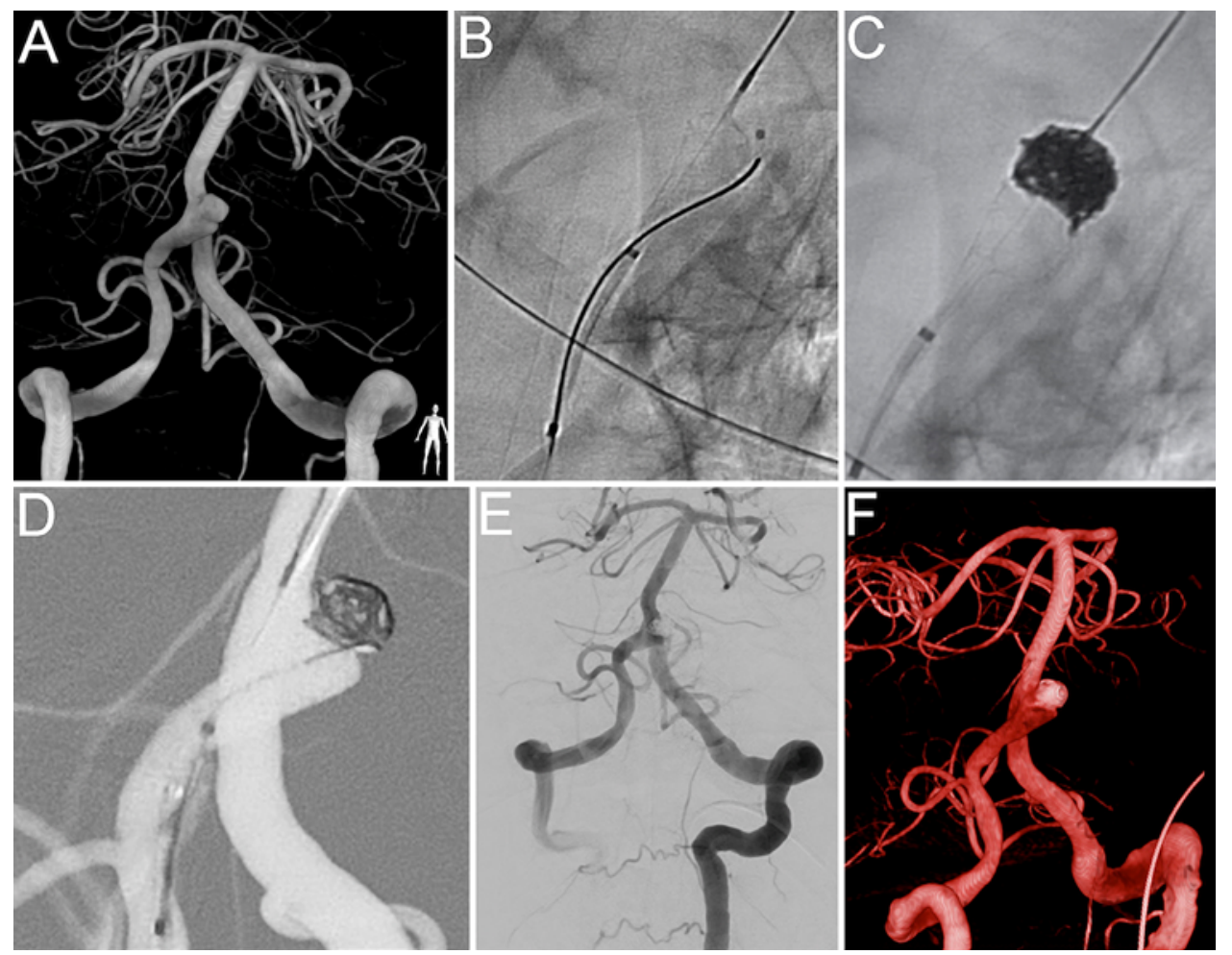

FIG. 3. Case 3. Angiographic imaging of a ruptured VA aneurysm. A: 3D rotational angiography showing an aneurysm on the $\mathrm{V}_{4}$ segment of the right VA. B: Unsubtracted imaging of Cascade placement into the aneurysm sac. C: Intraprocedural DSA (posterior-anterior view) showing coiling of the aneurysm. D: Intraprocedural DSA (posterior-anterior view) showing accurate positioning of the remodeling-net device. E and F: Postprocedure DSA showing complete occlusion of the aneurysm (MRRC type II) with suppression of the coil signal (E) and without suppression of the coil signal (F). Figure is available in color online only.

in the parent artery during inflation. However, this finding should be confirmed in larger randomized controlled studies comparing the rate of thromboembolism when performing the net-assisted remodeling technique with standard BRT. A potential disadvantage of the nonocclusive net-assisted remodeling technique is that it is impossible to immediately protect the vessel in the event of rupture, and to prevent coil prolapse while recapturing the device. However, no ruptures were experienced and no coil mass prolapsed while the Cascade device was recaptured in this study. If coil prolapse were to occur, the device could be reinflated to navigate the microcatheter, the number of coils could be increased, and a stent inserted if necessary, as is the case when using the BRT. The maintenance of blood flow would not add time pressure to the interventionalist during this rescue procedure.

The periprocedural use of antiplatelet therapy is commonly recommended to minimize thromboembolic complications in patients undergoing neuroendovascular procedures, ${ }^{4,8}$ although there is no consensus regarding the optimal regimen. The antiplatelet regimen is inferred from the cardiology literature without large clinical trials to support it in patients treated with neuroendovascular procedures. ${ }^{3}$ This was the first experience using this device for the net-assisted remodeling technique, and we could not decide the antiplatelet regimen preoperatively in the absence of data on its use in conjunction with this procedure. We judged it reasonable to maintain postprocedural antithrombotic therapy according to the standard practice in each center after a coiling procedure, which takes into account the clinical characteristics of the patients. Interestingly, 2 patients with a ruptured aneurysm were treated during the acute phase only with heparin, with no periprocedural antiplatelet therapy, and they experienced no thromboembolic complications. We suggest that randomized studies should be performed to test whether adjunctive antiplatelet therapy can be reduced in patients treated with the Cascade device to assist endovascular coil embolization in the acute phase of subarachnoid hemorrhage.

The Comaneci device (Rapid Medical) was the first device proposed for the remodeling-net technique with a variable cell size diameter of $1.33 \mathrm{~mm}^{2}$ that would allow the entanglement of a small coil. ${ }^{6}$ In a retrospective study of 14 aneurysms treated with the Comaneci device, immediate, complete aneurysm occlusion was achieved in $64.3 \%$ of cases, whereas $35.7 \%$ had a remaining neck remnant. ${ }^{5} \mathrm{~A}$ follow-up of 11 cases (median 4.8 months) showed that $81.8 \%$ experienced complete occlusion and $18.2 \%$ achieved near-complete occlusion. ${ }^{5}$ The Cascade net device was purposefully developed to have a higher cell mesh density to achieve a cell size $<0.03 \mathrm{~mm}^{2}$ (a smaller area than the diameter of the currently available coils used for intracranial aneurysm embolization) to reduce the risk 


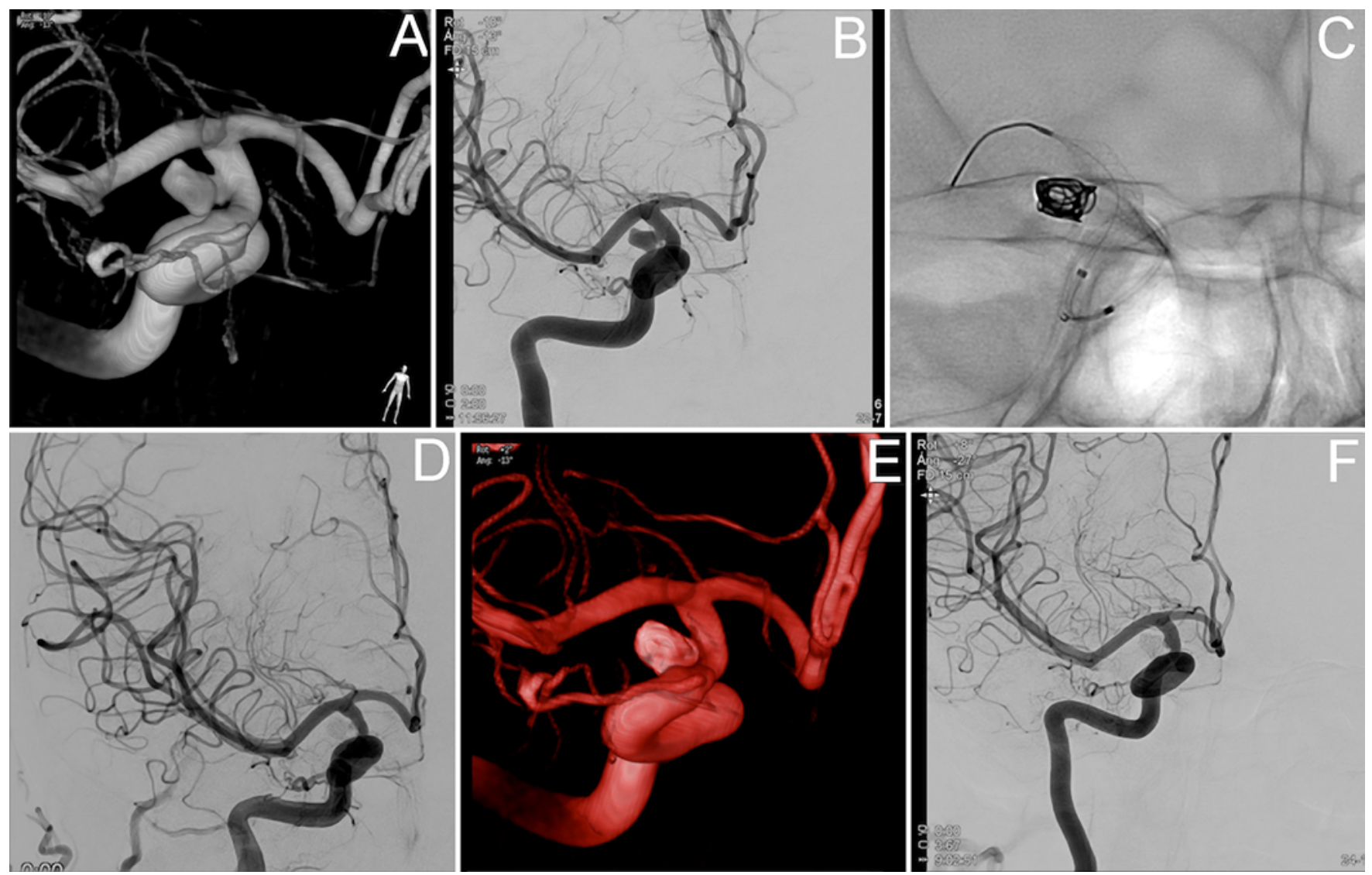

FIG. 4. Case 4. Angiographic imaging of ruptured ICA aneurysm. A: 3D rotational angiography showing an aneurysm on the posterior communicating segment of the right ICA. B: Preprocedural DSA (posterior-anterior view). C: Unsubtracted imaging of Cascade placement into the parent vessel and coiling of the aneurysm neck. D and E: Postprocedural DSA (D) and 3D rotational angiography (E) showing complete occlusion of the aneurysm. F: DSA image taken at the 6-month follow-up, confirming total occlusion (MRRC type I). Figure is available in color online only.

of coil herniation and coil entanglement. Moreover, the Cascade device has superior compliance and permits optimal neck coverage even in cases of complex anatomical relationship between the neck and vessel. Finally, the Cascade device includes a handle, which allows adjustment of the braided net diameter, providing an expected straight foreshortening of the device and adaptation of the radial force. In this way, the braided net responds predictably to operator manipulations.

The Cascade net device has several physical advantages compared to previous-generation devices. The responsive braided net provides excellent compliance with complex vessel geometry, allowing for superior aneurysm coverage even in challenging cases. The adjustable radial force permits a dynamic relationship between the intrasaccular microcatheter and the coils, making it possible to reorient the microcatheter as it is being stabilized, without blocking it. Moreover, the number and configuration of wires provide high compliance, providing a straight, noncontorted shape with a balance between rigidity and flexibility. This configuration and compliance allow for device manipulation for complex neurovascular conditions. All of these features may have contributed to the high rate of complete occlusion (MRRC type I in $73.3 \%$ of cases) and may have reduced the risk of thromboembolic complications in our series.

The mean duration of Cascade net use in this study was 30 minutes, but the duration of the entire procedure was similar to that of procedures performed without the device. ${ }^{3}$ Thus, while the absence of flow arrest alleviates time pressure on the clinician during coiling, this does not increase the overall procedure time.

\section{Limitations of the Study}

A major limitation of this study is the lack of a control arm and the inclusion of a small number of patients, particularly the group with ruptured aneurysms. The relatively short duration of follow-up, along with the few patients with reported results for follow-up, may have resulted in the underestimation of complication events or deterioration of MRRC type. Moreover, the different antiplatelet regimens used may have introduced variability in the results. Future prospective randomized studies are needed to define the best antiplatelet strategy in patients treated with the net-assisted remodeling technique. In particular, the possibility of reducing the need for antiplatelet therapy in patients with ruptured aneurysms should be tested. 


\section{Conclusions}

Our initial experience suggests that the Cascade net device may be safe and effective for assisting endovascular coil embolization of intracranial aneurysms. Based on our experience, the main advantage of the Cascade is that it helps the clinician by alleviating the time pressure associated with balloon-assisted coiling to reduce the risk of thromboembolism during the procedure, while its compliance permits optimal neck coverage. The Cascade device presents a further technical refinement of the remodeling technique that may increase the success rate of the procedure and expand the application of this technique in small aneurysms with complex anatomy, with better control in technically challenging cases. ${ }^{14}$ Further studies are needed to confirm possible extended indications for the Cascade net device for complex aneurysms and other conditions.

\section{Acknowledgments}

We acknowledge Superior Medical Experts for editing assistance.

\section{References}

1. Albayram S, Selcuk H, Kara B, Bozdag E, Uzma O, Kocer N, et al: Thromboembolic events associated with balloon-assisted coil embolization: evaluation with diffusion-weighted MR imaging. AJNR Am J Neuroradiol 25:1768-1777, 2004

2. Brinjikji W, Lanzino G, Cloft HJ, Rabinstein A, Kallmes DF: Endovascular treatment of very small ( $3 \mathrm{~mm}$ or smaller) intracranial aneurysms: report of a consecutive series and a meta-analysis. Stroke 41:116-121, 2010

3. de Gast AN, Soepboer A, Sluzewski M, van Rooij WJ, Beute GN: How long does it take to coil an intracranial aneurysm? Neuroradiology 50:53-56, 2008

4. Farrokh S, Owusu K, Lara LR, Nault K, Hui F, Spoelhof B: Neuro-interventional use of oral antiplatelets: a survey of neuro-endovascular centers in the United States and review of the literature. J Pharm Pract [epub ahead of print], 2019

5. Fischer S, Weber A, Carolus A, Drescher F, Götz F, Weber $\mathrm{W}$ : Coiling of wide-necked carotid artery aneurysms assisted by a temporary bridging device (Comaneci): preliminary experience. J Neurointerv Surg 9:1039-1097, 2017

6. Gupta R, Kolodgie FD, Virmani R, Eckhouse R: Comaneci neck bridging device for the treatment of cerebral aneurysms. J Neurointerv Surg 8:181-185, 2016

7. Hope JK, Byrne JV, Molyneux AJ: Factors influencing successful angiographic occlusion of aneurysms treated by coil embolization. AJNR Am J Neuroradiol 20:391-399, 1999

8. Kim KS, Fraser JF, Grupke S, Cook AM: Management of antiplatelet therapy in patients undergoing neuroendovascular procedures. J Neurosurg 129:890-905, 2018

9. Lawson AL, Chandran A, Puthuran M, Goddard T, Nahser $\mathrm{H}$, Patankar T: Initial experience of coiling cerebral aneurysms using the new Comaneci device. BMJ Case Rep 2015:bcr2015011726, 2015
10. Moret J, Cognard C, Weill A, Castaings L, Rey A: The "remodelling technique" in the treatment of wide neck intracranial aneurysms. Angiographic results and clinical follow-up in 56 cases. Interv Neuroradiol 3:21-35, 1997

11. Pierot L, Cognard C, Anxionnat R, Ricolfi F, CLARITY Investigators: Remodeling technique for endovascular treatment of ruptured intracranial aneurysms had a higher rate of adequate postoperative occlusion than did conventional coil embolization with comparable safety. Radiology 258:546553, 2011

12. Pierot L, Cognard C, Spelle L, Moret J: Safety and efficacy of balloon remodeling technique during endovascular treatment of intracranial aneurysms: critical review of the literature. AJNR Am J Neuroradiol 33:12-15, 2012

13. Pierot L, Spelle L, Leclerc X, Cognard C, Bonafé A, Moret $\mathrm{J}$ : Endovascular treatment of unruptured intracranial aneurysms: comparison of safety of remodeling technique and standard treatment with coils. Radiology 251:846-855, 2009

14. Piotin M, Blanc R: Balloons and stents in the endovascular treatment of cerebral aneurysms: vascular anatomy remodeled. Front Neurol 5:41, 2014

15. Spiotta AM, Bhalla T, Hussain MS, Sivapatham T, Batra A, Hui F, et al: An analysis of inflation times during balloonassisted aneurysm coil embolization and ischemic complications. Stroke 42:1051-1055, 2011

16. Stapleton CJ, Torok CM, Rabinov JD, Walcott BP, Mascitelli JR, Leslie-Mazwi TM, et al: Validation of the Modified Raymond-Roy classification for intracranial aneurysms treated with coil embolization. J Neurointerv Surg 8:927-933, 2016

\section{Disclosures}

Dr. Tomasello is a proctor and consultant for Medtronic, Stryker, MicroVention, and Perflow. This research did not receive any specific grant from funding agencies in the public, commercial, or not-for-profit sectors.

\section{Author Contributions}

Conception and design: Tomasello, Hernandez, Gramegna, Aixut, Barranco Pons, Jansen, Zawadzki, Lopez-Rueda, Parra-Fariñas, Piñana, Dinia, Arikan, Rovira. Acquisition of data: all authors. Analysis and interpretation of data: all authors. Drafting the article: Tomasello, Gramegna, Dinia. Critically revising the article: Tomasello, Gramegna. Reviewed submitted version of manuscript: all authors. Statistical analysis: Tomasello, Gramegna. Administrative/technical/material support: Gramegna. Study supervision: Tomasello, Gramegna.

\section{Correspondence}

Alejandro Tomasello: Vall d'Hebron University Hospital, Barcelona, Spain. alejandro.tomasello@idi.gencat.cat. 